\title{
Special Trilinear Functions with Few Applications
}

\author{
Aesha A. Juroud, Bashir W. Sharif, Ali M. Awin \\ Department of Mathematics, University of Tripoli, Tripoli, Libya \\ Email: awinsus@yahoo.com
}

How to cite this paper: Juroud, A.A. Sharif, B.W. and Awin, A.M. (2021) Special Trilinear Functions with Few Applications. Journal of Applied Mathematics and Physics, 9, 2698-2705.

https://doi.org/10.4236/jamp.2021.911173

Received: September 24, 2021

Accepted: November 7, 2021

Published: November 10, 2021

Copyright $\odot 2021$ by author(s) and Scientific Research Publishing Inc. This work is licensed under the Creative Commons Attribution International License (CC BY 4.0).

http://creativecommons.org/licenses/by/4.0/

(c) (i) Open Access

\begin{abstract}
Special bilinear functions (SBF) proved to be applicable in many situations and for a good number of problems. Hence it is important to generalize them to a higher degree by expanding previous work. In the beginning, we give a quick review of SBF [or quacroms of second degree and dimension $2 \times n$ ]; then we give a few applications based on previously published research concentrating on their use in evaluating some special functions and where we present the evaluation of Chebyshev polynomials as a new work. Following that, we define special trilinear functions (STF) of three $n$-tuples vectors, which are the generalization of SBF. Finally, a few applications, such as taking the product of three polynomials of degree $n$, are given stressing the fact that the process of taking the product of three integers using STF techniques, practically, takes place in a very efficient way and with no mentioned effort. A short discussion on the future of the subject constitutes the conclusion of our article.
\end{abstract}

\section{Keywords}

Bilinear, Trilinear, Function, Vectors, Polynomial, Special, Quacrom

\section{Introduction}

Special bilinear functions (SBF) of two real vectors were introduced, with the name "quacroms of dimension $2 \times n$ " [1]. The applications presented, then, were taking the product of two polynomials of degree $n$ and hence taking the product of two integers, reflecting the efficiency of the process in practice. Representing various quantities in the fields of mathematics and physics was the next useful thing to obtain [2].

More applications were found for them, especially after introducing linear quacrom equations (LQE) along with their solutions, where new algorithms were designed to compute known special functions, or polynomials, such as Bernoulli 
polynomials, Hermite polynomials, and Legendre polynomials [3] [4].

A generalization to quacroms of dimension $3 \times n$ was also introduced and studied with few applications [5].

In the next section, we give a quick review of SBF, adding an extra example of its use to calculate the first few Chebyshev polynomials. In the section to follow, we present special trilinear functions (STF) (or quacroms of dimension $3 \times n$ ) but with a new face, expanding the subject so as to clarify the concepts and to get a deeper insight. In Section 4, we present a few applications for STF. Finally, we present a brief discussion.

\section{A quick Review of SBF}

\section{Definition 1}

Consider a real-valued function $f$ of a real-valued matrix $A=\left(\begin{array}{cccc}a_{1} & a_{2} & \cdots & a_{n} \\ b_{1} & b_{2} & \cdots & b_{n}\end{array}\right)$, or of a pair of $n$-vectors $\boldsymbol{a}=\left(a_{1}, a_{2}, \cdots, a_{n}\right)$ and $\boldsymbol{b}=\left(b_{1}, b_{2}, \cdots, b_{n}\right)$, with the following properties

i) $f\left(k_{1} \boldsymbol{a}, k_{2} \boldsymbol{b}\right)=k_{1} k_{2} f(\boldsymbol{a}, \boldsymbol{b}), k_{1}$ and $k_{2}$ are scalars.

ii) $f(\boldsymbol{a}, \boldsymbol{b}+\boldsymbol{c})=f(\boldsymbol{a}, \boldsymbol{b})+f(\boldsymbol{a}, \boldsymbol{c})$ and $f(\boldsymbol{a}+\boldsymbol{b}, \boldsymbol{c})=f(\boldsymbol{a}, \boldsymbol{c})+f(\boldsymbol{b}, \boldsymbol{c})$.

iii) $f\left(\boldsymbol{e}_{i}, \boldsymbol{e}_{j}\right)=\delta_{i+j, n+1}$, where $\boldsymbol{e}_{i}$ is the $t^{\text {th }}$ unit vector, then, $f$ is the SBF (or quacrom) of $A$ written as

$$
\left.f(\boldsymbol{a}, \boldsymbol{b}) \equiv Q_{2 \times n}(A)=\right] \begin{array}{cccc}
a_{1} & a_{2} & \cdots & a_{n} \\
b_{1} & b_{2} & \cdots & b_{n}
\end{array}
$$

where $Q_{2 \times n}(A)$ is of second degree and $\mathrm{n}^{\text {th }}$ order, or of dimension $2 \times \mathrm{n}$ [4].

Note that the SBF has a good number of algebraic properties, e.g. $Q_{2 \times n}(A)$ represents the convolution of the two vectors $\boldsymbol{a}$ and $\boldsymbol{b}$. Moreover, it has proved to be useful in many applications. To mention one is the computation of the product of two polynomials and hence the efficient way of evaluating the product of two integers [4].

\section{Example 1}

To compute

i) $]_{5}^{2}\left[\right.$, ii) $\begin{array}{ll}2 & 1 \\ 5 & 3\end{array}[$, iii) $] \begin{array}{l}1 \\ 3\end{array}[$, iv) $21 \times 53$

Using SBF techniques.

To solve we see that

i) $]_{5}^{2}[=2 \times 5=10$

ii) $\begin{array}{ll}2 & 1 \\ 5 & 3\end{array}[=2 \times 3+1 \times 5=11$

iii) $] \begin{aligned} & 1 \\ & 3\end{aligned}[=1 \times 3$

iv) $21 \times 53=\left(\begin{array}{l}2 \\ 5\end{array}\right)\left(\begin{array}{ll}2 & 1 \\ 5 & 3\end{array}\right)()\left(\begin{array}{l}1 \\ 3\end{array}\right)=(10)(11)(3)=1113$. 


\section{Example 2}

Using SBF to calculate the product $214 \times 536$.

To calculate the product using SBF procedure, we see that

$$
\begin{aligned}
& \left.\begin{array}{lll}
2 & 1 & 4 \\
5 & 3 & 6
\end{array}=(] \begin{array}{l}
2 \\
5
\end{array}\right)\left(\begin{array} { l l } 
{ 2 } & { 1 } \\
{ 5 } & { 3 }
\end{array} [ ) \left(\begin{array}{lll}
2 & 1 & 4 \\
5 & 3 & 6
\end{array}[)\left(\begin{array}{ll}
1 & 4 \\
3 & 6
\end{array}[)(] \begin{array}{l}
4 \\
6
\end{array}\right)\right.\right. \\
& =(2 \times 5)(2 \times 3+1 \times 5)(2 \times 6+5 \times 4+1 \times 3)(1 \times 6+3 \times 4)(4 \times 6) \\
& =(10)(11)(35)(18)(24)=(11)(4)(7)(0)(4)=114704
\end{aligned}
$$

Note that the solutions are shown here in a formal way, but in practice, the process takes place in a very efficient manner and the result is written in one line. The brackets represent the decimal position $10^{i}, i=0,1,2,3, \cdots$.

Another important application for SBF is its use in computing some special functions such as Bernoulli, Hermite, and Legendre polynomials [3] [4]; in the following subsection, we present an algorithm to calculate the first few Chebyshev polynomials using SBF techniques.

\section{The Use of SBF to Compute Chebyshev Polynomials}

To proceed with the computation, we need to introduce the linear SBF equation (LQE) which is of the form

$$
] \begin{array}{ccccccc}
a_{1} & \cdots & a_{i} & \cdots & a_{j} & \cdots & a_{n} \\
b_{1} & \cdots & x & \cdots & b_{j} & \cdots & b_{n}
\end{array}=c(i+j=n+1)
$$

where the $a^{\prime} s$, the $b$ 's, and $c$ are real.

Equation (2) is equivalent to the equation $a x=b$; this leads to an interesting application keeping in mind the definition and properties of SBF.

As mentioned before, using this equation and SBF Bernoulli, Hermite, and Legendre polynomials were calculated in a simple and straightforward manner

[3] [4]. Here, we give another example of using Equation (2) and SBF procedure; namely, the computation of the first few Chebyshev polynomials [6].

Now, Equation (2) can be simplified by dividing the elements of the first row (and c) by $a_{j}$ and put in the form

$$
] \begin{array}{ccccccc}
a_{1} & \cdots & a_{i} & \cdots & 1 & \cdots & a_{n} \\
b_{1} & \cdots & x & \cdots & b_{j} & \cdots & b_{n}
\end{array}=c(i+j=n+1)
$$

where the new a's and $c$ are the old ones divided by $a_{j}$. The solution then is clearly

$$
x=] \begin{array}{ccccccc}
-a_{1} & \cdots & -a_{i} & \cdots & 1 & \cdots & -a_{n} \\
b_{1} & \cdots & c & \cdots & b_{j} & \cdots & b_{n}
\end{array}[(i+j=n+1)
$$

\section{Example 3}

To solve the LQE $] \begin{array}{lll}4 & 2 & 6 \\ 1 & x & 3\end{array}[=10$, we see that 2$] \begin{array}{lll}2 & 1 & 3 \\ 1 & x & 3\end{array}[=10$ which implies that $] \begin{array}{lll}2 & 1 & 3 \\ 1 & x & 3\end{array}[=5$; and hence 
$x=] \begin{array}{ccc}-2 & 1 & -3 \\ 1 & 5 & 3\end{array}[=-2 \times 3+1 \times 5-3 \times 1=-4$.

We proceed, now, with the calculation of Chebyshev polynomials.

The generating function for Chebyshev polynomials $\left(T_{n}(x)\right)$ is given by [7]

$$
g(x, t)=\frac{1-x t}{1-2 x t+t^{2}}=\sum_{n=0}^{\infty} T_{n}(x) t^{n}
$$

This equation can be written as

$$
\frac{1-x t}{1-2 x t+t^{2}}=\sum_{n=0}^{j} T_{n}(x) t^{n}+t^{j+1} \frac{F(t)}{1-2 x t+t^{2}}
$$

The right-hand side of Equation (6) can be simplified to get

$$
\frac{\sum_{n=0}^{j} T_{n}(x) t^{n}-2 x \sum_{n=0}^{\infty} T_{n}(x) t^{n+1}+\sum_{n=0}^{\infty} T_{n}(x) t^{n+2}+t^{j+1} F(t)}{1-2 t x+t^{2}}
$$

with a few algebraic simplifications the numerator of (7) is written as

$$
\begin{aligned}
& ] \begin{array}{c}
1 \\
T_{0}(x)
\end{array}[+] \begin{array}{cc}
1 & -2 x \\
T_{0}(x) & T_{1}(x)
\end{array}[t+] \begin{array}{ccc}
1 & -2 x & 1 \\
T_{0}(x) & T_{1}(x) & T_{2}(x)
\end{array}\left[t^{2}+\cdots\right. \\
& +] \begin{array}{ccc}
1 & -2 x & 1 \\
T_{k-2}(x) & T_{k-1}(x) & T_{k}(x)
\end{array}\left[t^{k}+\cdots, k \geq 2\right.
\end{aligned}
$$

Using (7), (8), and Equation (6) and comparing coefficients of $t^{n}$, we get

$$
\begin{gathered}
] T_{0}(x)[=1, \\
] \begin{array}{cc}
1 & -2 x \\
T_{0}(x) & T_{1}(x)
\end{array}[=-x, \\
{\left[\begin{array}{ccc}
1 & -2 x & 1 \\
T_{0}(x) & T_{1}(x) & T_{2}(x)
\end{array}[=0\right.}
\end{gathered}
$$

In general, we obtain

$$
] \begin{array}{ccc}
1 & -2 x & 1 \\
T_{k-2}(x) & T_{k-1}(x) & T_{k}(x)
\end{array}[=0, k \geq 2
$$

Solving the first three LQE's, we get

$$
\left.T_{0}(x)=\right] \begin{aligned}
& 1 \\
& 1
\end{aligned}\left[=1, \quad T_{1}(x)=\right] \begin{array}{cc}
1 & 2 x \\
1 & -x
\end{array}\left[=2 x-x=x, \text { and } T_{2}(x)=\right] \begin{array}{ccc}
1 & 2 x & -1 \\
1 & x & 0
\end{array}\left[=2 x^{2}-1\right.
$$

Note that the rest of the polynomials can, easily, be obtained in the same manner. Moreover, if we consider the general LQE in Equation (12), we see that its solution is given by

$$
\left.T_{k}(x)=\right] \begin{array}{ccc}
1 & 2 x & -1 \\
T_{k-2}(x) & T_{k-1}(x) & 0
\end{array}\left[=2 x T_{k-1}(x)-T_{k-2}(x), k \geq 2\right.
$$

Hence, we have

$$
T_{k}(x)=2 x T_{k-1}(x)-T_{k-2}(x), k \geq 2
$$

This is of course a very good recurrence relation, obtained via SBF techniques, 
and which enables us to compute all Chebyshev polynomials once we know $T_{0}(x)$ and $T_{1}(x)$.

\section{Special Trilinear Functions}

\section{Definition 2}

If $\boldsymbol{a}=\left(a_{1}, a_{2}, \cdots, a_{n}\right), \quad \boldsymbol{b}=\left(b_{1}, b_{2}, \cdots, b_{n}\right), \quad \boldsymbol{c}=\left(c_{1}, c_{2}, \cdots, c_{n}\right)$ are $n$-vectors; consider all real-valued functions $f^{l}(\boldsymbol{a}, \boldsymbol{b}, \boldsymbol{c})$ such that

i) $f^{l}\left(k_{1} \boldsymbol{a}, k_{2} \boldsymbol{b}, k_{3} \boldsymbol{c}\right)=k_{1} k_{2} k_{3} f^{l}(\boldsymbol{a}, \boldsymbol{b}, \boldsymbol{c})$

ii) $f^{l}(\boldsymbol{a}+\boldsymbol{b}, \boldsymbol{c}, \boldsymbol{d})=f^{l}(\boldsymbol{a}, \boldsymbol{c}, \boldsymbol{d})+f^{l}(\boldsymbol{b}, \boldsymbol{c}, \boldsymbol{d})$ and all other possible combinations.

iii) $f^{l}\left(\boldsymbol{e}_{i}, \boldsymbol{e}_{j}, \boldsymbol{e}_{k}\right)=\delta_{i+j+k, 2 n+2-l}$, where $\boldsymbol{e}_{i}$ is the $i^{\text {th }}$ unit vector and $l=1,2, \cdots, n$.

then $f^{l}(\boldsymbol{a}, \boldsymbol{b}, \boldsymbol{c})$ is the $I^{\text {th }}$ kind TLF of $A=\left(\begin{array}{lll}a_{1} & \cdots & a_{n} \\ b_{1} & \cdots & b_{1} \\ c_{1} & \cdots & c_{1}\end{array}\right)$ of the third degree and $n^{\text {th }}$ order, or simply of dimension $3 \times n$ [5], and is denoted by

$$
f^{l}(\boldsymbol{a}, \boldsymbol{b}, \boldsymbol{c}) \equiv Q_{3 \times n}^{l}(\boldsymbol{a}, \boldsymbol{b}, \boldsymbol{c})=\mid \begin{array}{ccc}
a_{1} & \cdots & a_{n} \\
b_{1} & \cdots & b_{n} \\
c_{1} & \cdots & c_{n}
\end{array}{ }_{l}
$$

\subsection{Few Lemmas}

\section{Lemma 1}

$\boldsymbol{a}=0, \boldsymbol{b}=0$, or $\boldsymbol{c}=0$ implies that $f^{l}(\boldsymbol{a}, \boldsymbol{b}, \boldsymbol{c})=0$.

The proof of this lemma is a direct consequence of (i) of the definition 2.

Lemma 2

$$
Q_{3 \times n}^{l}(\boldsymbol{a}, \boldsymbol{b}, \boldsymbol{c})=\sum_{i=1}^{l-1} c_{n-i+1} Q_{2 \times(n-l+i)}\left(A_{1, n-l+i}\right)+\sum_{i=l}^{n} c_{i-l+1} Q_{2 \times i}\left(A_{n-i+1, n}\right)
$$

\section{Proof}

From definition 2 one can see that

$$
Q_{3 \times n}^{l}(\boldsymbol{a}, \boldsymbol{b}, \boldsymbol{c})=\sum_{k} c_{k} \sum_{i, j} a_{i} b_{j} f^{l}(\boldsymbol{a}, \boldsymbol{b}, \boldsymbol{c})=\sum_{k} c_{k_{k}} \sum_{i, j} a_{i} b_{j} \delta_{i+j+k, 2 n+2-l}
$$

Hence we get

$$
Q_{3 \times n}^{l}(\boldsymbol{a}, \boldsymbol{b}, \boldsymbol{c})=\sum_{k} c_{k}\left(\sum_{i, j} a_{i} b_{j} \delta_{i+j, 2 n+2-l-k}\right)
$$

Equation (19) is the required result, expressed in Equation (17) since $k$ in the summation can be divided into two parts $k=1, l-1$ and $k=l, n$ and evaluating the various terms in the sum.

\subsection{Properties}

1) From lemma 2 it is a direct consequence that $Q_{3 \times n}^{l}(\boldsymbol{a}, \boldsymbol{b}, \boldsymbol{c})$ can be written as

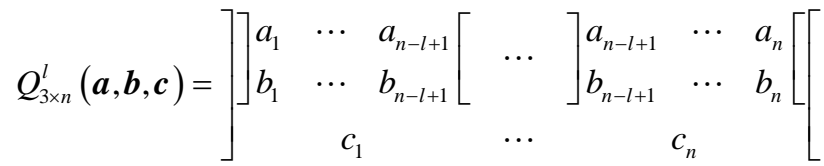


This implies that $Q_{3 \times n}^{l}(\boldsymbol{a}, \boldsymbol{b}, \boldsymbol{c})$ is a SBF of the second degree and $n^{\text {th }}$ order.

2) From the previous property, it is clear that rows are also interchangeable for STF of dimension $3 \times n$. This can easily be verified by an example.

3) If $\boldsymbol{\beta}=k \boldsymbol{a}$ then $Q_{3 \times n}^{l}(\boldsymbol{\beta}, \boldsymbol{b}, \boldsymbol{c})=k Q_{3 \times n}^{l}(\boldsymbol{a}, \boldsymbol{b}, \boldsymbol{c})$.

4) Evaluating $Q_{3 \times n}^{l}(\boldsymbol{a}, \boldsymbol{b}, \boldsymbol{c})$ implies that we make a transition in the following manner

$$
Q_{2 \times l}\left(A_{n-l+1, n}\right) \rightarrow Q_{2 \times n}\left(A_{1, n}\right) \rightarrow Q_{2 \times(n-l+1)}\left(A_{1, n-l+1}\right)
$$

\subsection{Few Applications}

$$
\begin{aligned}
& \text { If } f(x)=\sum_{i=1}^{n} a_{i} x^{n-i}, g(x)=\sum_{i=1}^{n} b_{i} x^{n-i}, h(x)=\sum_{i=1}^{n} c_{i} x^{n-i} \text {, then } \\
& \left.\left.\left.f(x) g(x) h(x)=x^{3 n-3}\right] \begin{array}{l}
a_{1} \\
b_{1} \\
c_{1}
\end{array}{ }_{1}+x^{3 n-4}\right] \begin{array}{ll}
a_{1} & a_{2} \\
b_{1} & b_{2} \\
c_{1} & c_{2}
\end{array}{ }_{2}+\cdots+x^{2 n-2}\right] \begin{array}{lll}
a_{1} & \cdots & a_{n} \\
b_{1} & \cdots & b_{n} \\
c_{1} & \cdots & c_{n}
\end{array}{ }_{n} \\
& +x^{2 n-1}\left[\begin{array}{ccc}
a_{1} & \cdots & a_{n} \\
b_{1} & \cdots & b_{n} \\
c_{1} & \cdots & c_{n}
\end{array}{ }_{n-1}+\cdots+x^{n-1}\left[\begin{array}{ccc}
a_{1} & \cdots & a_{n} \\
b_{1} & \cdots & b_{n} \\
c_{1} & \cdots & c_{n}
\end{array}{ }_{2}\right.\right. \\
& \left.\left.\left.+x^{n-2}\right] \begin{array}{ccc}
a_{2} & \cdots & a_{n} \\
b_{2} & \cdots & b_{n} \\
c_{2} & \cdots & c_{n}
\end{array}{ }_{1}+\cdots+x^{1}\right] \begin{array}{ll}
a_{n-1} & a_{n} \\
b_{n-1} & b_{n} \\
c_{n-1} & c_{n}
\end{array} L_{1}+x^{0}\right] \begin{array}{l}
a_{n} \\
b_{n} \\
c_{n}
\end{array} L_{1}
\end{aligned}
$$

Again for the product of two integers we take $=10$.

\section{Example 4}

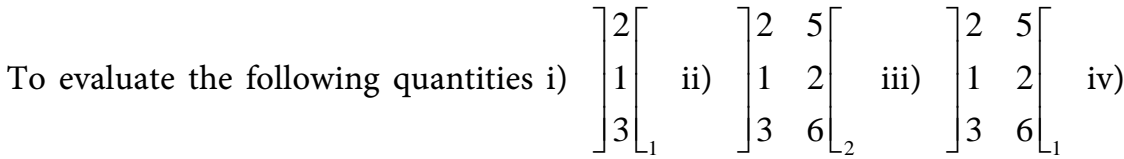
] $\begin{aligned} & 5 \\ & 2 \\ & 6\end{aligned}[$ and then to compute the product $25 \times 12 \times 36$

We see from the properties of STF that

$$
\begin{aligned}
& \text { i) } \begin{array}{l}
2 \\
1 \\
3
\end{array}\left[\begin{array}{l}
2 \times]_{1}^{2} \\
1
\end{array}[=3 \times(2 \times 1)=6\right. \\
& \text { ii) } \begin{array}{ll}
2 & 5 \\
1 & 2 \\
3 & 6
\end{array}{ }_{2}=3 \times \begin{array}{ll}
2 & 5 \\
1 & 2
\end{array}[+6 \times] \begin{array}{l}
2 \\
1
\end{array}[=3(2 \times 2+5 \times 1)+6(2 \times 1)=27+12=39 \\
& \text { iii) } \begin{array}{ll}
2 & 5 \\
1 & 2 \\
3 & 6
\end{array}\left[\begin{array}{l}
1 \\
1
\end{array}\right] \begin{array}{ll}
2 & 5 \\
1 & 2
\end{array}[+3] \begin{array}{l}
5 \\
2
\end{array}[=6(2 \times 2+5 \times 1)+3(5 \times 2)=54+30=84 \\
& \text { iv) } \begin{array}{l}
5 \\
2 \\
6
\end{array}\left[\begin{array}{l}
1 \\
1
\end{array}\right] 2\left[\begin{array}{l}
5 \\
2
\end{array}=6 \times 5 \times 2=60\right.
\end{aligned}
$$


v)

$$
\begin{aligned}
25 \times 12 \times 36 & =\begin{array}{rl}
2 & 5 \\
1 & 2 \\
3 & 6
\end{array}\left(\begin{array}{l}
6 \\
3 \\
3
\end{array}\right)\left(\begin{array}{l}
2 \\
1
\end{array}\right)\left(\begin{array}{ll}
7 & 5 \\
1 & 2 \\
3 & 6
\end{array} L_{2}\right)\left(\begin{array}{ll}
2 & 5 \\
1 & 2 \\
3 & 6
\end{array} L_{1}\right)\left(\begin{array}{l}
5 \\
2 \\
6 \\
L_{1}
\end{array}\right) \\
& =(6)(39)(84)(60)=(10)(8)(0)(0)=10800
\end{aligned}
$$

Therefore using STF techniques for multiplying three numbers we obtained the required result in a beautiful and efficient way. In fact, the process with, a certain simple arrangement, can be accomplished with just a pencil and a piece of paper in one line.

\section{Example 5}

To compute the product $123 \times 456 \times 789$, we need to evaluate the various STF quantities. This is done as follows

First we compute the STF's

a) $\begin{aligned} & 1 \\ & 4 \\ & 7\end{aligned}[=7 \times] \begin{aligned} & 1 \\ & 4\end{aligned}[=7(1 \times 4)=28$.

b) $\begin{array}{ll}1 & 2 \\ 4 & 5 \\ 7 & 8\end{array}[=7 \times] \begin{array}{ll}1 & 2 \\ 4 & 5\end{array}[+8 \times] \begin{aligned} & 1 \\ & 4[\end{aligned}$

$$
=7(1 \times 5+2 \times 4)+8(1 \times 4)=123
$$

$$
] \begin{array}{lll}
1 & 2 & 3 \\
4 & 5 & 6 \\
7 & 8 & 9
\end{array}{ }_{3}=7 \times\right] \begin{array}{ccc}
1 & 2 & 3 \\
4 & 5 & 6
\end{array}[+8 \times] \begin{array}{cc}
1 & 2 \\
4 & 5
\end{array}[+9 \times] \begin{gathered}
1 \\
4
\end{gathered}
$$

c)

$$
=7(1 \times 6+3 \times 4+2 \times 5)+8(1 \times 5+2 \times 4)+9(1 \times 4) .
$$

$$
7 \begin{array}{lll}
1 & 2 & 3 \\
4 & 5 & 6 \\
7 & 8 & 9
\end{array}\left\lfloor_{2}=7 \times\right] \begin{array}{ll}
2 & 3 \\
5 & 6
\end{array}[+8] \begin{array}{lll}
1 & 2 & 3 \\
4 & 5 & 6
\end{array}[+9] \begin{array}{ll}
1 & 2 \\
4 & 5
\end{array}
$$

d)

$$
=7(2 \times 6+3 \times 5)+8(1 \times 6+3 \times 4+2 \times 5)+9(1 \times 5+2 \times 4) .
$$

$$
=189+224+117=530
$$

$$
] \begin{array}{lll}
1 & 2 & 3 \\
4 & 5 & 6 \\
7 & 8 & 9
\end{array} L_{1}=7 \times\right] \begin{aligned}
& 3 \\
& 6
\end{aligned}\left[+8 \times \begin{array}{ll}
2 & 3 \\
5 & 6
\end{array}[+9 \times] \begin{array}{ccc}
1 & 2 & 3 \\
4 & 5 & 6
\end{array}[\right.
$$

e)

$$
\begin{aligned}
& =7(3 \times 6)+8(2 \times 6+3 \times 5)+9(1 \times 6+3 \times 4+2 \times 5) . \\
& =126+216+252=594
\end{aligned}
$$

f) $\begin{array}{ll}2 & 3 \\ 5 & 6 \\ 8 & 9\end{array}\left[\begin{array}{l}1 \\ 1\end{array}=8 \times \begin{array}{l}3 \\ 6\end{array}\left[+9 \times \begin{array}{ll}2 & 3 \\ 5 & 6\end{array}[\right.\right.$

$$
=8(3 \times 6)+9(2 \times 6+3 \times 5)=144+243=387
$$

g) $\begin{aligned} & 3 \\ & 6 \\ & 9\end{aligned}\left[\begin{array}{l}3 \\ 1\end{array}=9 \times \begin{array}{l}3 \\ 6\end{array}=9(3 \times 6)=162\right.$.

Therefore our product is given by 


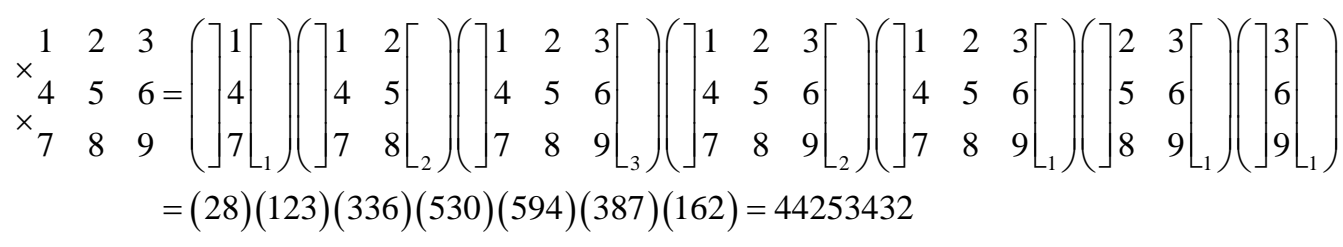

\section{A Brief Discussion}

We have seen how the generalization from SBF to STF has taken place. Applications of STF so far were few but future studies will reveal more applications for them. Moreover, we believe that the generalization to a higher dimension, $4 \times n$ and higher, is a challenging target. This will be a possible future work.

We note also the resemblance between quacroms and determinants where we see that in evaluating $Q_{3 \times n}(A)$ we have to evaluate various $Q_{2 \times n}(A)$, and the same thing happens in evaluating determinants where evaluating cofactors is needed to fully evaluate a certain determinant. However, we should note that there are differences between the two: the first difference is that quacroms deal with a rectangular array of numbers while determinants consist of $n^{2}$ numbers; the second one is the difference in the method of evaluation of each one of them.

\section{Conflicts of Interest}

The authors declare no conflicts of interest regarding the publication of this paper.

\section{References}

[1] Awin, A.M. (1982) Quacroms and Their Applications. International Journal of Mathematical Education in Science and Technology, 13, 93-97. https://doi.org/10.1080/0020739820130113

[2] Awin, A.M. (1985) Few More Applications for Quacroms. Libyan Journal of Science, 14, 49-54.

[3] Awin, A.M. (2014) On the Use of Special Bilinear Functions to Compute Bernoulli Polynomials. International Journal of Computers and Technology, 14, 5337-5342. https://doi.org/10.24297/ijct.v14i1.2120

[4] Awin, A.M. (2015) The Use of Special Bilinear Functions in Computing Some Special Functions. Journal of Progressive Research in Mathematics, 5, 437-443.

[5] Awin, A.M. (1995) Algebra of Quacroms. Technical Report, ICTP, IC/95/189.

[6] Juroud, A.A. (2016) Special Bilnear Functions and Their Applications. MSc. Thesis, University of Tripoli, Tripoli.

[7] Arfken, G. (1985) Mathematical Methods for Physicists. 3rd Edition, Academic Press, Orlando. 